\title{
LA COMUNICACIÓN COMO HERRAMIENTA ESTRATÉGICA EN LA INDUSTRIA DE LA MODA
}

\section{COMMUNICATION AS A STRATEGIC TOOL IN THE FASHION INDUSTRY}

\section{AUTORES}

José Luis Del Olmo Arriaga

Universitat Abat Oliba CEU. Barcelona (España)

jlolmo@uao.es

\section{Joan Francesc Fondevila}

Universitat Abat Oliba CEU. Barcelona (España)

jffondevila@ilimit.es

\section{RESUMEN}

La comunicación es uno de los instrumentos de marketing más importantes que utilizan los diseñadores y las empresas de moda de cualquier sector y tamaño. Todo lo que éstos hacen tiene un papel fundamental en el proceso de comunicación: el mensaje del propio producto, el precio, la colección, el estilismo, el desfile, la presentación en el punto de venta, la feria, la información que transmite la fuerza de ventas, etc.; de hecho, el producto moda ya comunica por sí mismo.

Para que la moda sea aceptada y consumida, debe ser, en primer lugar, conocida y seguida, y ello sólo será posible si se conoce y comunica. No se puede seguir una tendencia o comprar una prenda si el cliente o consumidor no tiene conciencia de la marca o del producto. Para ello, en este estudio se analizan los medios de comunicación en la moda, a través de los cuales las organizaciones del sector son capaces de influir y de establecer una comunicación eficaz con la demanda del producto moda.

\section{PALABRAS CLAVE}


Comunicación - Marketing - Moda - Estrategia - Industria

\section{ABSTRACT}

Communication is one of the most important marketing tools used by designers and companies in the fashion industry. Everything they do has a critical role in the communication process: the message of the product itself, the price, the collection, styling, the parade, the presentation at the point of sale, the fair, the force transmitting information sales, etc. in fact, fashion and communicates the product itself.

For that fashion is accepted and consumed, it should be, first, known and followed, and this will only be possible if you know and communicate. Can not follow a trend or buy an item if the customer or consumer is not aware of the brand or product. For this reason, this study examines the communication tools in fashion, through which sector organizations will be able to communicate effectively and influence demand.

\section{KEY WORDS}

Communication - Marketing - Fashion - Strategy - Industry

\section{ÍNDICE}

1. La elección de los medios de comunicación

1.1 Revistas

1.2. Prensa

1.3. Televisión

1.4. Cine

1.5. Radio 
1.6. Publicidad exterior

1.7. Catálogo de moda

1.8. Comunicación en el punto de venta

1.9. Marketing directo

1.10. Comunicación a través del showroom

1.11. Desfiles de moda

1.12. Ferias

1.13. Internet

1.14. Directorios o anuarios profesionales

1.15. House Organ o Newsletter.

1.16. Videos de moda

1.17. Relaciones públicas

1.18. El sponsoring

1.19. Patrocinio y mecenazgo

1.20. Otras formas de comunicación

2. Conclusiones

\section{La elección de los medios de comunicación}

En la elección de los medios de comunicación los operadores del sector de la moda deben concretar dónde, cómo y cuándo comunicar sus productos, tendencias y colecciones. En sectores de gran consumo (por ejemplo, jeans, vestuario deportivo, etc.) se utilizan medios de largo alcance, mientras que en mercados muy segmentados (pret-à-porter de lujo, por ejemplo) se utilizan instrumentos de comunicación más personales, como las revistas especializadas de moda. Se pueden utilizar distintos medios y soportes simultáneamente con objeto de aprovechar mejor las posibilidades de cada medio y el efecto de sinergia entre ellos. Para su elección 
se deben valorar, por ejemplo, a qué medios están expuestos los destinatarios, si el medio sirve para difundir el mensaje o el coste de la operación ${ }^{1}$.

Es habitual definir la comunicación según los medios utilizados; así, se distingue la comunicación convencional y la no convencional:

- Comunicación convencional. Contempla la utilización de medios de comunicación masivos como revistas, prensa, radio, cine, etc.

- Comunicación no convencional. Se denomina también below de line y está constituida por un variado número de técnicas de promoción alternativas a la comunicación publicitaria tradicional (desfiles, ferias, promociones de ventas, patrocinio, etc.).

En la elección de los medios se debe tener en cuenta la cantidad de información a transmitir, la duración del mensaje, el público objetivo al que se dirige la comunicación, el tipo de canal de distribución, el ámbito geográfico de actuación, las características y cobertura de cada medio, el número de contactos, así como el coste del contacto útil.

Dentro de los medios de comunicación cabe considerar las revistas, la prensa, la televisión, el cine, la radio, la publicidad exterior, los catálogos de moda, la comunicación en el punto de venta, el marketing directo, la comunicación a través del showroom, los desfiles de moda, las ferias, Internet, los directorios o anuarios profesionales, los House Organ o Newsletter, los videos de moda, las relaciones públicas, el sponsoring y el patrocinio.

\section{1. Revistas}

\footnotetext{
${ }^{1}$ En España existen diferentes organismos que proporcionan información sobre audiencias, cobertura y costes de cada medio 0 soporte; por ejemplo, OJD (Oficina de Justificación de la Difusión), EGM (Estudio General de Medios), SOFRES, etc.
} 
Es uno de los medios más utilizados por las grandes empresas del sector, debido a su carácter propio para dar a conocer las tendencias de moda. Este tipo de medio permite reproducir anuncios con una gran calidad de impresión. Sin embargo, dada la audiencia limitada de la mayor parte de soportes, los costes por impacto son bastante elevados.

En función del perfil del lector, las revistas pueden ser de ámbito general, revistas especializadas en moda y publicaciones profesionales del sector.

Revistas de ámbito general. Se trata de un medio de comunicación de carácter generalista, a través del cual se puede contactar con receptores comunes (consumidores), mediante un mensaje de moda adecuado a este público. Es útil para el lanzamiento o promoción de productos de gran difusión, dado que permite alcanzar un gran número de lectores.

Revistas de moda especializadas y orientadas al consumidor final. Este medio de comunicación dispone de audiencias sensibles a la moda. La oferta se presenta de forma atractiva y amena para el lector. Presenta un contenido diversificado, según los ámbitos que caracterizan la oferta del producto (vestuario en general, vestuario masculino, femenino, infantil, de ceremonia, prenatal, vestuario íntimo, piel, etc.). El tipo de mensaje que transmite se dirige a un tipo de lector más o menos experto en moda. Las ediciones de primavera (febrero/marzo) y de otoño (agosto/septiembre) contienen amplios editoriales, y a menudo se publican suplementos que muestran las colecciones internacionales que se han presentado tres meses antes en París, Milán, Londres y Nueva York. (tabla 1). Sólo las grandes firmas del sector y diseñadores con amplia capacidad económica planifican sus campañas de comunicación en este tipo de medios, dado los altos costes de inserción de los anuncios en relación a las grandes tiradas de algunas de las revistas de moda presentes en el mercado. 

comunicación como herramienta estratégica en la industria de la moda. No 18. Marzo. Año XIII. Páginas: 1-30

ISSN: 1576-3420 DOI: http://dx.doi.org/10.15198/seeci.2009.18.1-30

Tabla 1. Principales revistas de moda dirigidas al consumidor final.

\begin{tabular}{|c|c|c|}
\hline $\begin{array}{l}\text { Tipo de } \\
\text { vestuario }\end{array}$ & País & Revista \\
\hline \multirow{7}{*}{$\begin{array}{l}\text { Vestuario } \\
\text { femenino }\end{array}$} & España & $\begin{array}{l}\text { Burda, Cosmopolitan, Elle, Glamour, Marie Claire, Ragazza, Telva, } \\
\text { Vogue, Woman, etc. }\end{array}$ \\
\hline & Francia & $\begin{array}{l}\text { Collections, Elle, Jardin des Modes, Joyce, L'Officiel de la Couture et de } \\
\text { la mode de Paris, Madame, Marie Claire, Vogue, etc. }\end{array}$ \\
\hline & Italia & Donna, Harper's Bazaar, Linea Italiana, Lei, Vogue, etc. \\
\hline & Alemania & Burda International, Fashion Guide, Madam, Vogue, etc. \\
\hline & Inglaterra & Elle, Bella, British Vogue, Harper's and Queene, Prima, etc. \\
\hline & $\begin{array}{l}\text { Estados } \\
\text { Unidos }\end{array}$ & $\begin{array}{l}\text { Allure, Burda, Cosmopolitan, Elle, Essence, Glamour, Harper's Bazaar, } \\
\text { Madame, Mademoiselle, Seventeen, Mirabelle, Vogue, etc. }\end{array}$ \\
\hline & Japón & Fashion Yearbook, Hi Fashion, Mode et Mode, Madam, Mrs, etc. \\
\hline \multirow{5}{*}{$\begin{array}{l}\text { Vestuario } \\
\text { masculino }\end{array}$} & Francia & L'Officiel Hommes, Vogue Hommes, etc. \\
\hline & Italia & $\begin{array}{l}\text { Linea Italiana Uomo, Uomo Vogue, Uomo Harper's Bazaar, Uomo Mare, } \\
\text { Per Lui, etc. }\end{array}$ \\
\hline & Alemania & Männer Vogue, etc. \\
\hline & $\begin{array}{l}\text { Estados } \\
\text { Unidos }\end{array}$ & $\begin{array}{l}\text { Daily News Record (DNR), EM (Ebony Man), Esquire, GQ (Gentelman } \\
\text { Quarterly), "Man", etc. }\end{array}$ \\
\hline & Japón & Dansen, Men's Club, Mr., etc. \\
\hline \multirow{2}{*}{$\begin{array}{l}\text { Vestuario } \\
\text { niño/niña }\end{array}$} & Italia & Donna Bambini, Moda Bimbi, Vogue Bambini, etc. \\
\hline & Japón & Baby Fashion, Sesame, etc. \\
\hline \multirow{4}{*}{$\begin{array}{l}\text { Vestuario } \\
\text { Nupcial }\end{array}$} & España & Novias, Sposabella, Vogue Sposa, etc. \\
\hline & Francia & Mariages, etc. \\
\hline & Italia & Sposa, Vogue Sposa, etc. \\
\hline & \begin{tabular}{|l} 
Estados \\
Unidos
\end{tabular} & Bridal Guide, Bride's, Modern Bride, etc. \\
\hline
\end{tabular}

Fuente: Elaboración propia.

Las revistas disponen, en general, de dos formatos diferentes de anuncio publicitario: el publirreportaje y el anuncio convencional. 
Publirreportaje. Se distingue del anuncio convencional por su similitud con la información periodística habitual de la revista. Desde el punto de vista del contenido, se caracteriza porque en su redactado no se suele utilizar un tono persuasivo directo, como ocurre con el anuncio de pago. Es utilizado frecuentemente dentro de los suplementos o páginas especiales de carácter monográfico o sectorial (por ejemplo, para comunicar el avance de una nueva temporada de moda) que, de forma periódica, publican las revistas de moda o los suplementos dominicales de la prensa diaria.

- $\quad$ Anuncio convencional. Se trata de un anuncio publicitario pagado por el anunciante, cuyo contenido está básicamente limitado por el formato contratado y por las características técnicas del soporte.

Una modalidad de comunicación publicitaria complementaria al anuncio de pago en las revistas especializadas de moda está constituida por el denominado modelo italiano, el cual parte del principio de negociar sistemáticamente la publicación de fotos informativas a cambio de páginas de publicidad. Desde 1980, este sistema ha tenido cada vez más adeptos, especialmente en Estados Unidos, lo que permite un gran control por parte de las empresas y de los diseñadores, ya que al inicio de cada temporada pueden predecirse, sin temor a equivocarse, su nivel de divulgación e influir también en las prendas que se muestran.

El anunciante negocia, por ejemplo, la aparición de 40 modelos en la información editorial a cambio de la inserción publicitaria de 80 modelos. La efectividad de esta táctica deja al editor impotente, ya que se ve obligado a no reflejar su propio gusto y su elección en el estilismo, para mostrar sólo la estrategia comercial de los anunciantes. Este sistema de negociación impidió la combinación de prendas de distintos diseñadores durante la mayor parte de la década de los años ochenta, período del total look. 


\subsection{Prensa}

La prensa es, normalmente, el medio de comunicación más inmediato que se hace eco de la información concerniente a la moda, a los desfiles de los diseñadores, a las tendencias de moda, etc. La comunicación de este tipo de eventos puede ser interesante para el consumidor y para el canal de distribución, tanto si su contenido es redaccional como si es de carácter publicitario.

Se trata de un medio eficaz, que tiene un efecto inmediato aunque de vida limitada. Publicaciones internacionales como Le Monde 0 el New York Times tienen una enorme influencia sobre el gran público, aunque su utilización exige el empleo de agencias de publicidad o de relaciones públicas especializadas, cuya función consiste en crear, producir y planificar las campañas de promoción de la moda.

Se pueden distinguir dos tipos de soportes: los diarios de pago y las publicaciones gratuitas:

- Diarios. Publican diariamente información general, económica, cultural, etc. Según su alcance, tiene una cobertura nacional, regional o local y suele incluir suplementos, entre ellos el dominical, el cual incluye, generalmente, una sección de moda, con reportajes a todo color de tendencias, desfiles, etc.

- $\quad$ Publicaciones gratuitas. Son de periodicidad diaria o variable y se distribuyen de forma personalizada entre el público. Este tipo de soportes ha experimentado un notable incremento en publicidad y en difusión, sobre todo en áreas geográficas locales. 


\subsection{Televisión}

A pesar de su alto poder de atracción y elevada audiencia, la televisión es un medio publicitario por el cual apuestan muy pocas empresas de moda. Supone unos costes absolutos elevados y su eficacia se diluye cada vez más debido al bajo poder de segmentación, proliferación de canales, saturación de anuncios y al hábito del efecto zapping. Sólo grandes marcas como Levi's o Nike, y grandes almacenes, como El Corte Inglés, planifican sus campañas en este medio. Sin embargo, algunas de las principales firmas de Alta Costura, pret-a-porter de lujo y diseñadores utilizan este medio para promocionar sus líneas de perfumes con objeto de capitalizar su imagen de marca.

\section{4. Cine}

Es un medio que se caracteriza por una gran selectividad de la audiencia, así como una gran capacidad de impacto visual y auditivo que lo hace atractivo para las grandes marcas de moda de gran consumo. Sin embargo, tiene como principales inconvenientes la audiencia limitada y un alto coste por impacto.

\section{5. Radio}

Se caracteriza por provocar un efecto rápido a un coste relativamente bajo. Además, presenta la ventaja de la selectividad geográfica y demográfica de clientes potenciales. Por ello, es un medio apropiado para los establecimientos detallistas de moda, para que puedan anunciar acontecimientos extraordinarios como inauguraciones o promociones. Sin embargo, la falta de apoyo visual reduce la efectividad y permanencia de los mensajes publicitarios en este medio. 


\section{6. Publicidad exterior}

La publicidad exterior puede realizarse por medio de vallas, cabinas telefónicas, transporte público, así como mobiliario urbano (marquesinas, opys, quioscos de prensa, etc.). Es un medio publicitario que tiene una gran flexibilidad geográfica, aunque su efectividad está condicionada a la ubicación del soporte. Muchas empresas de gran difusión utilizan este medio publicitario dentro de sus campañas de publicidad local. Su mayor inconveniente es el coste, dado que para ser eficaz debe cubrir un amplio territorio geográfico durante un período mínimo de quince días, lo que resulta difícil para las medianas y pequeñas empresas del sector.

\section{7. Catálogo de moda}

Es otro instrumento de comunicación tradicional entre fabricante, canal de distribución y consumidor final. Se puede utilizar de forma conjunta con las revistas especializadas de moda, con el marketing directo y con carteles o displays enviados a los puntos de venta, de forma que provoque una imagen coordinada y una sinergia entre ambos tipos de comunicación.

El folleto de las colecciones estacionales tiene como objeto informar y poner al día al cliente de forma puntual; éste debe ser realizado para las diferentes estaciones (primavera-verano, otoño-invierno) y contener toda la información necesaria, con el apoyo de fotografías que lo enriquezcan visualmente; además, para quien desee efectuar un pedido debe figurar toda la información complementaria necesaria (nombre y código del modelo, color, talla, descripción del tejido, observaciones 
técnicas, precio, condiciones de pago, pedido mínimo, condiciones de venta, fecha de servicio, etc.).

\section{8. Comunicación en el punto de venta}

Este tipo de comunicación agrupa un conjunto de técnicas que permiten poner de relieve el producto en el lugar de venta. Se trata de una comunicación orientada fundamentalmente al consumidor; en efecto, a través de los escaparates del comercio detallista y de todos aquellos instrumentos de comunicación que se pueden utilizar en su interior (carteles, maniquíes, displays, videos de desfiles, etc.), el consumidor recibe una ayuda eficaz en su decisión de compra.

Para que un producto moda tenga éxito en el mercado éste debe mostrarse de forma atractiva en el punto de venta; en este sentido, el canal de distribución tiende a poner más de relieve en el punto de venta aquellos productos y marcas que orientan su comunicación hacia el punto de venta. Ello se confirma, sobre todo, en los puntos de venta en régimen de autoservicio, donde el consumidor efectúa un acto de compra no asistida; por tanto, cualquier reclamo de comunicación, sonoro o visual, puede influir de forma decisiva en su elección de un producto o marca concreta.

Los objetivos que persigue la comunicación en el punto de venta pueden ser de diferente naturaleza:

- Apoyar una campaña de publicidad o de promoción, haciendo que la marca esté presente en el punto de venta.

Animar las ventas, de forma complementaria a otras acciones comerciales.

Constituye el último elemento recordatorio que tiene ante sí el cliente antes del acto de compra. Contribuye a la presentación del producto, informa al consumidor sobre 
nuevos artículos y, sobre todo, favorece la compra por impulso. Dispone de múltiples medios y soportes: presentación de las colecciones, desfiles, carteles, displays, expositores, pancartas, soportes porta-folletos, vídeos, escucha de mensajes sonoros, distribución de materiales ilustrativos, etc.

\subsection{Marketing directo}

El rápido desarrollo de las técnicas de comunicación ha contribuido, en gran medida, al desarrollo del marketing directo como un instrumento estratégico de marketing al servicio de la empresa de moda. Esta técnica de marketing comprende un conjunto de actividades por las que se dirige una comunicación personalizada y en un breve espacio de tiempo a aquellos destinatarios a los que el producto que ofrece la empresa les puede interesar. Utiliza uno o más medios de comunicación publicitaria, con objeto de provocar una respuesta medible o incluso una venta inmediata; al contrario de lo que sucede con la comunicación general que informa sobre el producto y alcanza a una audiencia de masas.

El marketing directo ofrece un conjunto de ventajas ${ }^{2}$ que favorecen su rápida expansión, pues permite ser adoptado por todo tipo de empresas en el sector de la moda:

- $\quad$ se dirige a un público objetivo preciso, a través del uso de bases de datos de clientes o de listas externas,

establece una comunicación interactiva con el cliente, que permite, al mismo tiempo, conseguir ventas,

- los resultados son medibles de forma clara y directa, permite crear y mantener una base de datos de clientes,

- facilita tanto un canal controlado de comunicación como de distribución,

- $\quad$ permite establecer estrategias o tácticas invisibles para los competidores.

\footnotetext{
${ }^{2}$ Alet, J. (1994). Marketing Directo Integrado. Gestión 2000. Barcelona.
} 
Los ámbitos de actuación del marketing directo son numerosos y abarcan las acciones que se pueden realizar sobre la propia red de ventas, el canal de distribución o el consumidor final del producto. Ello significa que la empresa puede enviar al consumidor final, por ejemplo, un mailing personalizado que incluya el catálogo de moda de la temporada, con objeto de apoyar al canal de distribución (tráfico en el punto de venta, concursos de ventas, etc.), o una invitación para un desfile, una feria o un showroom. También puede distribuir una revista corporativa a mayoristas, detallistas, centrales de compra, etc. a través del correo para crear una imagen favorable entre los diferentes miembros del canal. Su mayor dificultad es el coste y la obtención de la información necesaria para decidir el destinatario de la acción.

Una campaña de marketing directo puede incluir más de un medio de comunicación en la misma acción, dado que, en general, existe una compatibilidad entre los diversos medios; una combinación de varios de ellos (mailing, teléfono, revistas, etc.) resulta, en muchas ocasiones, más eficaz que uno sólo. Por ello, la empresa debe determinar los medios más adecuados con objeto conseguir que su oferta tenga interés para los clientes, así como contemplar los factores condicionantes, como el tipo de mercado, el producto o el sistema de distribución que la caracteriza.

Los medios que intervienen de forma más habitual en las acciones de marketing directo son el mailing y el telemarketing:

Mailing. Es uno de los medios más flexibles, personales y, por tanto, más utilizados en marketing directo. Aunque resulta el medio con el coste por impacto más caro, después del teléfono, desde el punto de vista de respuestas obtenidas se sitúa entre los mejores. Se trata de un mensaje personalizado enviado por correo a personas previamente seleccionadas, sin limitaciones de formato o de tiempo. Si este mercado no se selecciona correctamente la información se dirigirá a un público no interesado en lo que la empresa comunica, y los resultados de la acción serán nulos. 
Telemarketing. Este es el medio más inmediato de marketing directo. Se basa en un sistema de comunicación que utiliza el teléfono para conseguir una respuesta directa, de tal forma que, mediante un guión y a través de la comunicación telefónica, se procede a la información o concertación de una entrevista para la fuerza de ventas o incluso a la venta del producto o servicio. El contacto telefónico supone un apoyo o soporte de la comunicación realizada en otros medios (prensa, revistas, mailing, etc.); también puede apoyar a la fuerza de ventas en la cualificación de prospectos o en el tratamiento y seguimiento de visitas a los clientes.

\subsection{Comunicación a través del showroom}

El término show-room hace alusión a un desfile técnico en el que se muestra la verdadera colección o los modelos que estarán finalmente en los puntos de venta ${ }^{3}$. Se trata del lugar donde clientes e intermediarios del canal de distribución pueden ver la exposición de las colecciones ofrecidas durante las estaciones en curso, así como efectuar pedidos de las prendas que son de su interés para posteriormente poder venderlas en sus propios puntos de venta.

Una empresa puede exponer su propia gama de productos, pero, si el showroom pertenece a un distribuidor, mayorista o representante, éstos suelen exponer la oferta de productos de otras empresas o diseñadores a los cuales representan; en este caso, el surtido presentado no tiene un alto nivel de competencia, para no perjudicar a ninguna de las empresas representadas.

El showroom ofrece la posibilidad de presentar en cualquier momento, pero, sobre todo, en el periodo del lanzamiento estacional, todas las colecciones a los clientes (canal de distribución y consumidores); para ello, la oferta presentada debe estar integrada con el resto de instrumentos de comunicación y promoción (desfile, prensa, etc.).

\footnotetext{
${ }^{3}$ Scipione, D. (2002). La moda. Manuali di comunicazione Ellissi. Milán.
} 
En el showroom se debe facilitar al cliente la posibilidad de ver, tocar y hacer probar los modelos presentados. Una presentación realizada con profesionalidad puede ser más útil que un desfile y pueden obtenerse mejores resultados de venta, además de ofrecer un servicio más personalizado y orientado al cliente.

La ubicación del showroom puede constituir un factor condicionante del éxito de la comunicación y venta de la colección. Puede estar integrada dentro de la propia empresa $o$ en cualquier otra ciudad donde haya una alto potencial de clientes y sea fácilmente accesible.

\subsection{Desfiles de moda}

El desfile es el evento por excelencia de comunicación de la moda, además de un medio de promoción, dado que ofrece la posibilidad a los compradores de convencerse para sus propias compras. El ambiente, la escenografía, la música, el casting, la presentación, la organización los modelos o los productos presentados constituyen aspectos que proporcionan un soporte perfecto a este vehículo de comunicación y de promoción. Es precisamente un desfile detrás de otro lo que hace construir la imagen de un diseñador y de una empresa frente al público y lo que permite ganan espacio en el mercado de la moda. No en vano, la prensa mundial acude a estas citas de la moda para elaborar noticias que no sólo servirán de cierre de los telediarios sino que también serán material para emisión de canales especializados ${ }^{4}$. Por ello, los desfiles de moda se situarían entre los muchos canales de comunicación de los que dispone el sector textil para generar una imagen corporativa. En este caso, hablaríamos de la confección de una imagen de frivolidad, tendente hacia la exclusividad de la que desde siempre se alimenta la moda ${ }^{5}$ (Herrero, 2004: 25).

\footnotetext{
${ }^{4}$ AA.VV. (2002). "Moda de España: punto de encuentro". Madrid, Sociedad Estatal para el Desarrollo del Diseño y la Innovación - DDI Ministerio de Economía, p. 163.

${ }^{5}$ Herrero, M. (2004): “Fascinación a la carta: La moda en la postmodernidad" en Codina, M. y Herrero, M. (2004). Mirando la moda: once reflexiones. Ediciones Internacionales Universitarias. Madrid.
} 
Los desfiles normalmente se realizan en los puntos de venta, en hoteles, dentro de un stand $o$ en un espacio adecuado en una feria, en el propio showroom o con ocasión de algún reportaje o espacio televisivo.

Un desfile debe poder alcanzar una serie de objetivos: informar y entretener al público sobre las nuevas colecciones, presentar los modelos de forma atractiva y sugerente, apoyar la decisión de compra de los compradores y afinar la oferta antes de la venta final. Para alcanzar estos objetivos se requiere una perfecta preparación del mismo; sólo así se podrá implicar al público asistente: periodistas de moda, compradores, clientes, consumidores, etc.

Como acontecimientos espectaculares, los desfiles pueden tener una fuerte repercusión en los medios de comunicación especializados, especialmente en las revistas de moda, sobre todo, por el acontecimiento periodístico que generan. Sin embargo, tienen el problema de su alto coste, por lo cual muchas empresas y diseñadores no pueden acceder a este medio de comunicación. Además, los compradores no disponen del tiempo suficiente para valorar de forma directa los productos presentados; ello hace necesaria su visita a los showroom con objeto de verificar personalmente las prendas que pueden ser de su interés.

Cada año tienen lugar en las capitales de la moda dos temporadas de desfiles: septiembre-octubre y marzo-abril. En los meses de otoño se presentan las colecciones de Alta Costura y prêt-a-porter de la temporada de primavera-verano del siguiente año y en primavera se presentan las de otoño-invierno. El calendario bianual de desfiles para compradores de prêt-à-porter ha pasado tradicionalmente de Londres a Milán, París y Nueva York, durante un período de cuatro semanas. Estos centros de diseño compiten entre sí por los clientes y compradores y por conseguir espacios de tiempo en el calendario internacional de los salones de moda. En España, las ciudades donde tienen lugar los principales desfiles de moda son Madrid (Pasarela Cibeles) y Barcelona (Salón Gaudí). 


\subsection{Ferias}

Son instrumentos de marketing que tienen como objetivo fundamental la comunicación, promoción y venta del producto moda a intermediarios del canal de distribución (en especial, los puntos de venta multimarca), dado que permiten establecer contacto con un gran número de clientes actuales y potenciales en un período de tiempo y espacio reducido. Los visitantes son, en su mayor parte, profesionales del sector: compradores, agentes, distribuidores, diseñadores y medios de comunicación, así como estilistas de tejidos y estilistas de confección, en general, que buscan información sobre tendencias de moda. La importancia de los diferentes salones viene determinada por dos parámetros: como ferias comerciales o como escaparates de estilo y tendencias ${ }^{6}$; por tanto, son un medio privilegiado que permite combinar la fuerza de ventas, la comunicación, la promoción, las relaciones públicas o la investigación de mercados.

Las ferias de moda están clasificadas según la oferta presentada: hombre, mujer, niño, novias, baño, lencería, complementos, etc. y tienen lugar en diferentes ciudades. La amplia oferta de ferias profesionales nacionales e internacionales (tabla 2), la tendencia a una mayor diversificación y especialización, la segmentación de expositores y visitantes, así como el constante encarecimiento de los costes globales de participación implican la necesidad de seleccionar y racionalizar los recursos destinados a la participación en las mismas ${ }^{7}$.

Antes de participar en una feria especializada, la empresa debe disponer de una serie de datos con objeto de determinar cuál interesa más para dar a conocer y promocionar sus productos. Esta información se puede obtener a través de las diversas fuentes que, directa 0 indirectamente están relacionadas con el salón y que conocen su historia y su desarrollo en anteriores ediciones (expositores, compradores

\footnotetext{
${ }^{6}$ I váñez, J. M. (2000). La gestión del diseño en la empresa. McGraw-Hill. Madrid.

${ }^{7}$ Le Monnier, F. (2000). Marketing Ferial. Gestión 2000. Barcelona.
} 

comunicación como herramienta estratégica en la industria de la moda. No 18. Marzo. Año XIII. Páginas: 1-30

ISSN: 1576-3420 DOI: http://dx.doi.org/10.15198/seeci.2009.18.1-30

directos, estilistas, diseñadores, usuarios finales, prensa especializada de moda, etc.).

Para asegurar el buen funcionamiento del evento y alcanzar los objetivos prefijados, es importante que la participación en la feria se programe y prepare de la mejor manera posible. Un óptimo recurso para apoyar la presencia en una feria es el de recurrir a la prensa genérica y especializada para divulgar la propia participación, así como las novedades que serán presentadas en la misma. Así mismo, la empresa o el diseñador deben hacer un esfuerzo de marketing y comunicación con objeto de transmitir la personalidad y la filosofía de la marca; para ello, un diseño atractivo del stand y un personal de venta competente y con capacidad de comunicarse con los diversos visitantes son instrumentos básicos para alcanzar el éxito.

Tabla 2. Principales ferias del sector de la moda en el circuito internacional.

\begin{tabular}{|c|c|c|c|}
\hline Mes & Sector & Denominación & Población \\
\hline \multirow{8}{*}{ Enero } & Vestuario Hombre & Pitti Immagine Uomo & Florencia (Italia) \\
\hline & Vestuario Mujer & Pret-à-Porter & París (Francia) \\
\hline & Vestuario Infantil & FIMI & Valencia (España) \\
\hline & Vestuario Infantil & Pitti Bimbo I & Florencia (Italia) \\
\hline & Moda & Hong Kong Fashion Week & Hong Kong (China) \\
\hline & Moda en Piel & Pielespaña & Barcelona (España) \\
\hline & Vestuario Íntimo y baño & Salon International de la Lingerie & París (Francia) \\
\hline & Hilados/Tejidos & Pitti Filati & Florencia (Italia) \\
\hline \multirow{8}{*}{ Febrero } & Vestuario Hombre y Mujer & London Fashion Week & Londres (Reino Unido) \\
\hline & $\begin{array}{l}\text { Vestuario Hombre, Mujer, } \\
\text { Íntimo y Baño }\end{array}$ & CPD Woman-Man I & Dusseldorf (Alemania) \\
\hline & Vestuario mujer & Pure Womenswear I & Londres (Reino Unido) \\
\hline & Vestuario mujer & Imagenmoda & Madrid (España) \\
\hline & Moda & Barcelona Fashion Week & Barcelona (España) \\
\hline & Moda & SIMM & Madrid (España) \\
\hline & Moda Pronta & Intermoda & Madrid (España) \\
\hline & Vestuario Infantil & Salon de la Mode Enfantine & París (Francia) \\
\hline
\end{tabular}


REVISTA DE LA SEECI.

Del Olmo Arriaga, José Luis y Fondevila, Joan Francesc (2009): La comunicación como herramienta estratégica en la industria de la moda. No 18. Marzo. Año XIII. Páginas: 1-30

ISSN: 1576-3420 DOI: http://dx.doi.org/10.15198/seeci.2009.18.1-30

\begin{tabular}{|c|c|c|c|}
\hline \multirow{7}{*}{ Marzo } & \begin{tabular}{|l} 
Vestuario \\
Prontomoda
\end{tabular} & Prêt-à-Porter & París (Francia) \\
\hline & Tejidos & Première Vision & París (Francia) \\
\hline & Vestuario infantil & $\mathrm{FIMI}$ & Valencia (España) \\
\hline & Calzado & GDS & Düsseldorf (Alemania) \\
\hline & Calzado & Modacalzado & Madrid (España) \\
\hline & Marroquinería y Piel & Iberpiel & Madrid (España) \\
\hline & Marroquinería & Mipel I & Milán (Italia) \\
\hline \multirow{5}{*}{ Abril } & Vestuario Mujer & $\begin{array}{ll}\text { American Designer Collection } \\
\text { Shows }\end{array}$ & Nueva York (Estados Unidos) \\
\hline & Vestuario Deportivo & Sportex & París (Francia) \\
\hline & Vestuario Profesional & IWWC & París (Francia) \\
\hline & Tejidos & Interstoff & Frankfurt (Alemania) \\
\hline & Moda y Accesorios & $\begin{array}{l}\text { Fall Fashion and Accesories } \\
\text { Market }\end{array}$ & Montreal (Canadá) \\
\hline \multirow{3}{*}{ Mayo } & Moda Gran Distribución & Intersélection & París (Francia) \\
\hline & Accesorios & $\begin{array}{l}\text { Fashion Accesories Expo and } \\
\text { Accesories Circuit }\end{array}$ & Nueva York (Estados Unidos) \\
\hline & Vestuario Íntimo & Intima Japan & Tokio (Japón) \\
\hline \multirow{6}{*}{ Junio } & Vestuario Hombre & Pitti Immagine Uomo & Florencia (Italia) \\
\hline & Vestuario infantil & Pitti Immagine Bimbo & Florencia (Italia) \\
\hline & Vestuario infantil & Kind + J ugend & Colonia (Alemania) \\
\hline & Hilados/Tejidos & Pitti Filati & Florencia (Italia) \\
\hline & Vestuario Hombre & NAMSB-Show & Nueva York (Estados Unidos) \\
\hline & Vestuario de Ceremonia & NoviaEspaña & Barcelona (España) \\
\hline \multirow{8}{*}{ Julio } & Vestuario Hombre & Designer Men's Wear Collections & París (Francia) \\
\hline & Vestuario Hombre & $\begin{array}{l}\text { SIHM-Salon Internacional de } \\
\text { I'Habillement Masculin }\end{array}$ & París (Francia) \\
\hline & Moda & Aute Couture & París (Francia) \\
\hline & Moda & Hong Kong Fashion Week & Hong Kong (China) \\
\hline & Vestuario Infantil & $\mathrm{FIMI}$ & Valencia (España) \\
\hline & Vestuario Infantil & Pitti Bimbo II & Florencia (Italia) \\
\hline & Vestuario Infantil & Mode Enfantine & París (Francia) \\
\hline & Moda & Imagen Moda & Madrid (España) \\
\hline
\end{tabular}


REVISTA DE LA SEECI.

Del Olmo Arriaga, José Luis y Fondevila, Joan Francesc (2009): La comunicación como herramienta estratégica en la industria de la moda. No 18. Marzo. Año XIII. Páginas: 1-30

ISSN: 1576-3420 DOI: http://dx.doi.org/10.15198/seeci.2009.18.1-30

\begin{tabular}{|c|c|c|c|}
\hline \multirow{6}{*}{ Agosto } & $\begin{array}{l}\text { Vestuario Hombre, Mujer, } \\
\text { Íntimo y Baño }\end{array}$ & CPD Woman-Man II & Dusseldorf (Alemania) \\
\hline & Vestuario mujer & Pure Womenswear II & Londres (Reino Unido) \\
\hline & Moda & SIMM & Madrid (España) \\
\hline & Moda de Vanguardia & The Edge & Las Vegas (Estados Unidos) \\
\hline & Moda & Salón de pequeños creadores & Londres (Reino Unido) \\
\hline & Calzado & WSA-Shoe Show & Las Vegas (Estados Unidos) \\
\hline \multirow{11}{*}{ Septiembre } & $\begin{array}{l}\text { Vestuario Mujer } \\
\text { Prontomoda }\end{array}$ & Prêt-à-Porter & París (Francia) \\
\hline & Moda & Barcelona Fashion Week & Barcelona (España) \\
\hline & Vestuario Íntimo y Baño & Lyon Mode City & Lyon (Francia) \\
\hline & Vestuario Íntimo & Fashion Cotery & Nueva York (Estados Unidos) \\
\hline & Marroquinería & Mipel II & Milán (Italia) \\
\hline & Calzado & Modacalzado & Madrid (España) \\
\hline & Calzado & GDS & Düsseldorf (Alemania) \\
\hline & Marroquinería y Piel & Iberpiel & Madrid (España) \\
\hline & Joyería y Relojería & Iberjoya & Madrid (España) \\
\hline & Bisutería y Complementos & Bisutex & Madrid (España) \\
\hline & Joyería & Bijoux & Milán (Italia) \\
\hline \multirow{5}{*}{ Octubre } & Vestuario Hombre y Mujer & London Fashion Week & Londres (Reino Unido) \\
\hline & Vestuario Infantil & Children's Club & Nueva York (Estados Unidos) \\
\hline & Accesorios & Première Classe & París (Francia) \\
\hline & Tejidos & Première Vision & París (Francia) \\
\hline & Tejidos & Lyontex & Lyon (Francia) \\
\hline \multirow{2}{*}{ Noviembre } & Moda Íntima & Intimate Apparel Salon & Nueva York (Estados Unidos) \\
\hline & Moda Gran Distribución & Intersélection & París (Francia) \\
\hline \multirow{2}{*}{ Diciembre } & Género de Punto & Moda Prima & Milán (Italia) \\
\hline & Calzado & FFANY & Nueva York (Estados Unidos) \\
\hline
\end{tabular}

Fuente: Elaboración propia, sobre base Textil Exprés.

\subsection{Internet}


Internet es un auténtico fenómeno de dimensión mundial con incidencia en todos los sectores empresariales, entre ellos el de la moda. La tecnología Internet se ha basado principalmente en los «escaparates» virtuales o catálogos electrónicos. Su filosofía consiste en la presentación de productos, marcas y empresas, aunque cada vez se desarrollan más proyectos en este medio con el objetivo de mejorar los canales de venta.

En la comunicación a través de Internet las empresas del sector de la moda pueden informar en tiempo real y a un bajo coste sobre cualquier oferta de productos, presentar las colecciones, las campañas de venta, etc. Este medio ofrece también la posibilidad de crear un catálogo electrónico cerrado, es decir, restringiendo el acceso a los usuarios que desee la empresa: representantes, clientes, distribuidores, mayoristas, etc. Esta solución puede ser de gran utilidad para facilitar la comunicación entre la empresa y sus agentes realizando una mínima inversión ${ }^{8}$.

Las fashion-websites de productores y diseñadores crean sus espacios on line para dar a conocer los desfiles más exclusivos a través de web-cams. Éstos son conscientes de que hoy en día lo que no está en Internet no existe ${ }^{9}$.

Con un objetivo más global se han incrementado los portales que ofrecen información, definen tendencias y permiten la compra on-line como, por ejemplo, bloomingdales.com, bluefly.com, ebay.com, eluxury.com, girlshop.com, etc. Entre ellos, destacan las diferentes ediciones digitales de Vogue en el mundo, como vogue.es en España o style.com (versión americana), auténticos iconos de la moda internacional en la red. Como espacio innovador merece destacar también themode.tv, una cadena de televisión on-line que está presente en pasarelas, inauguraciones y backstages de las capitales más importantes del mundo de la moda.

\footnotetext{
${ }^{8}$ Fundació Cetemmsa (2001). Resultados del estudio sobre el Impacto del Comercio Electrónico en el sector Textil Español. Edición propia. Barcelona.

${ }^{9}$ Contreras, M. (2002). "El nuevo templo de la moda" en revista Vogue Colecciones. Otoño/Invierno. P. 94-95.
} 
Las formas de comunicación que permite Internet son:

- Página web. Permite la presentación de la oferta (lanzamiento de nuevos productos, difusión de las colecciones, etc.), informa sobre actividades de la empresa (desfiles, participación en ferias, presentación en showroom, etc.) y apoya la imagen de la marca.

- Correo electrónico. Ofrece la posibilidad de establecer una comunicación abierta, inmediata y eficaz con el canal de distribución y con los consumidores (pedidos, solicitud de información, buzón de sugerencias, reclamaciones, etc.).

- $\quad$ Chat. Se puede dialogar en tiempo real con el canal de distribución y con el consumidor final.

Inserción de banners publicitarios. Consiste en un faldón publicitario con imágenes de producto, colección, etc. vinculadas a la página web de la empresa. Es la forma más popular de publicidad on-line.

- Comercio electrónico. Permite la transacción comercial on-line entre una empresa y un cliente de cualquier lugar del mundo, facilitando a éste su adquisición sin contacto físico.

A pesar de que el potencial de Internet es muy grande, también hay el peligro de que si la web no se promociona su eficacia comercial sea mínima. Por tanto, hay que promoverla en los diferentes «mercados» on-line del sector a nivel nacional e internacional y realizar una mayor o menor inversión en marketing, en función de la estrategia y del valor que la web suponga para la empresa ${ }^{10}$.

${ }^{10}$ Fundació Cetemmsa (2001). Resultados del estudio sobre el Impacto del Comercio Electrónico en el sector Textil Español. Edición propia. Barcelona. 


\subsection{Directorios o anuarios profesionales}

Los directorios o anuarios profesionales constituyen un medio de comunicación eficaz para dar a conocer la oferta de una empresa. Normalmente, fabricantes, miembros del canal de distribución, diseñadores, representantes, detallistas, etc. disponen de este medio como elemento de consulta para buscar o contactar con otras empresas del sector o para buscar cualquier nuevo proveedor. La presencia en este tipo de anuarios, bien sea a través de una reseña con los datos básicos de la empresa o mediante la inserción de un anuncio publicitario, puede aumentar la posibilidad de identificar y de contactar más fácilmente con la empresa anunciante. El mensaje que se transmite tiene la ventaja de que está presente durante un largo período de tiempo (aproximadamente, un año) para quien lo consulte; ello permite una comunicación permanente y eficaz durante el período de vigencia en el mercado. Este medio de comunicación es de carácter sectorial y suele solicitarse mediante suscripción (tabla 3).

Tabla 3. Principales directorios sectoriales del sector de la moda.

\begin{tabular}{|l|l|}
\hline País & Directorio \\
\hline España & Directorio sectorial. \\
\hline Inglaterra & Drapers Record, Textile View, View Colour, Wiewpoint, etc. \\
\hline Estados Unidos & $\begin{array}{l}\text { The Buyer's Blue Book, Davison's Textile Blue Book, Directory of Women's } \\
\text { and Children's Wear Speciality Stores, Garment Manufacturer's Index, } \\
\text { Nationwide Directory of Women's and Children's Wear Buyers. }\end{array}$ \\
\hline
\end{tabular}

Fuente: Elaboración propia.

\subsection{House Organ o Newsletter.}

Es una publicación breve que una empresa de determinado tamaño envía periódicamente a los clientes habituales y potenciales o al propio personal interno de 
la misma. Puede contener información sobre el lanzamiento de nuevos productos y colecciones, noticias sobre campañas de comunicación y de ventas o información variada sobre la empresa a un público interesado, por lo que resulta un eficaz instrumento de promoción y comunicación, así como un excelente potenciador de imagen.

\subsection{Vídeos de moda}

El vídeo constituye un instrumento de gran efecto en la comunicación de moda y puede utilizarse en muchos ámbitos como, por ejemplo, en la presentación de la empresa, de los productos, de la colección, de los desfiles realizados del diseñador, etc. Puede ser utilizado durante una entrevista con un cliente, en el showroom, en un punto de venta, durante una feria, también puede enviarse a los propios clientes o con ocasión de un seminario para la fuerza de ventas.

\subsection{Las relaciones públicas}

Las relaciones públicas son uno de los instrumentos de comunicación más importante en el sector de la moda y como tal apoya y complementa también al resto de instrumentos de la política de comunicación (publicidad, promoción, fuerza de ventas, etc.). Puede proporcionar a la empresa, a la marca o a sus productos una enorme difusión, conocimiento y notoriedad. Su valor diferencial es el de la credibilidad que conllevan sus acciones; al no dirigirse al comprador potencial y no tener un condicionante temporal, los receptores de los mensajes de relaciones públicas no siempre son conscientes de los contenidos persuasivos de estos mensajes, ya que no tiene el mismo valor un anuncio pagado que la opinión transmitida por un periodista de moda, cuya opinión es percibida como objetiva.

Las relaciones públicas persiguen generalmente relaciones de confianza que aporten a una empresa o marca el reconocimiento y apoyo de los clientes, sean actuales 0 
potenciales. El objetivo es, por tanto, establecer y desarrollar un clima de interés y de simpatía entre el público objetivo al que va dirigida la acción (empleados de la propia empresa, medios de comunicación, instituciones, miembros del canal de distribución, líderes de opinión, consumidores, etc.), mediante la utilización de técnicas de comunicación cargadas de persuasión. En cualquier caso, este instrumento de comunicación constituye una de las herramientas que más ha incrementado su presencia en las actividades de marketing de la moda en los últimos años.

Las actividades típicas de las relaciones públicas son las convocatorias, los comunicados o notas de prensa, las ruedas de prensa y las entrevistas:

Convocatoria. Consiste en la información de la celebración de un acontecimiento puntual: inauguración de una tienda, invitación a un desfile, presentación de una colección, etc.

- Comunicado o nota de prensa. Se trata de una información que se envía a los medios de comunicación del sector para comunicar las diferentes actividades de la empresa (desfile, presentación de la colección, lanzamiento de una nueva marca, etc.). Para ello, se debe confeccionar un dossier atractivo sobre la propia organización, sobre la marca o sobre los productos, en el cual se incluya suficiente material escrito y gráfico para los mass media. Se distinguen dos tipos:

- Nota previa. Es el anuncio del acontecimiento. Contiene, además de los datos básicos, los antecedentes, la documentación sobre el hecho noticioso y el eje de comunicación de la empresa. Se distingue de una convocatoria porque se elabora para ser publicada.

Nota resultante. Se elabora después de la noticia y debe destacar la información más relevante en una redacción periodística breve, clara y concisa. 
Rueda de prensa. Es una acción que debe reservarse para hechos noticiables que, por su extensión o contenido, merecen un tratamiento informativo superior al que proporciona una nota de prensa. Se convoca mediante un portavoz para dar cuenta de una determinada información a los medios de comunicación. Es una técnica poco utilizada en el sector de la moda; sólo las grandes compañías pueden proporcionar informaciones de interés que tengan como cauce informativo esta técnica de comunicación.

Entrevista. Consiste en una conversación personal para obtener, en exclusiva, información en profundidad sobre uno o varios temas. Todos los medios de comunicación, en especial la prensa especializada en moda, recoge habitualmente en sus páginas entrevistas con diseñadores reconocidos o con empresarios del sector. Diseñadores internacionales como Carolina Herrera y Giorgio Armani, o españoles como Ágata Ruiz de la Prada, Adolfo Domínguez o Custo Dalmau, han sabido capitalizar su imagen a través de esta interesante técnica de relaciones públicas. En cualquier caso, hay que saber cuándo y en qué condiciones se concede ese trato especial en los medios, porque no se debe «quemar» la imagen del entrevistado. También se debe preparar minuciosamente, elaborando un dossier para el medio de comunicación que facilite y enriquezca la reproducción de la entrevista. Si ésta es audiovisual hay que cuidar al máximo la imagen, las condiciones acústicas, el fondo para la televisión, etc.

\subsubsection{Diferentes estrategias de relaciones públicas en la moda}

Se pueden definir diferentes estrategias con las que concebir un acto de Relaciones Públicas en la moda. En primer lugar, se trata de proyectar la marca en el mercado, promoviendo una imagen positiva de la firma y siguiendo las pautas de todo evento 
de relaciones públicas para su ejecución ${ }^{11}$ (Fernández Souto, 2004: 24-25) en lo referido a:

Promover el respeto mutuo: entre los diseñadores, como promotores de tendencias y de "estilo" y la sociedad, seguidores de las modas pautadas por éstos,

La responsabilidad social: especialmente refiriéndonos a la responsabilidad social corporativa de las instituciones promotoras del evento, que con su presencia avalarían al pret-a-porter,

Armonizando los intereses públicos y privados: de tal manera que con el evento se promovería una imagen institucional de apoyo a la moda, y este hecho revertiría en los propios diseñadores, que verían promocionado su trabajo,

Promocionando productos y servicios, de tal manera que se atrajera personal cualificado y se promocionase la buena voluntad entre los empresarios y clientes,

Optimizando el beneficio: tanto tangible como intangible. $Y$ finalmente, Proyectando una imagen corporativa de los diseñadores españoles de mayor proyección.

\subsection{El sponsoring}

Es una forma de comunicación de una empresa basada en el apoyo financiero, en especies 0 en servicios a una actividad deportiva, con objeto de obtener un incremento de notoriedad o de un beneficio para su imagen. Se pueden esponsorizar personas, grupos y organizaciones deportivas. Para ello, se utiliza la comunicación o la promoción en relación con el acontecimiento esponsorizado. Por ejemplo, Hermenegildo Zegna esponsoriza competiciones de vela, Lacoste y Hugo Boss torneos de tenis internacionales, etc.

${ }^{11}$ Fernández Souto, A. B. (2002). Manual de programación e técnicas das rr.pp. Servizo de publicacións da Universidade de Vigo. Vigo. 


\subsection{Patrocinio y mecenazgo}

Son formas de apoyo a actividades sociales, culturales, etc., para favorecer la predisposición del público hacia los productos, marcas o la propia empresa. El patrocinador concede una prestación, fundamentalmente de valor económico, a un individuo, un acontecimiento, una institución, un medio de comunicación, etc. para que éste ponga en marcha una acción. No se trata de una prestación desinteresada, porque el patrocinado se obliga a algunas prestaciones hacia su benefactor, como, por ejemplo, lucir en su indumentaria el logotipo de la entidad que le apoya.

Un ejemplo de patrocinio lo constituye la Pasarela Cibeles que cuenta con el patrocinio de L'Oreal para la presentación de las colecciones de la moda española. La marca Loewe, a través de su Fundación, también apuesta por esta estrategia de comunicación convocando desde hace varios años un Concurso de Piano en Madrid. También ha tenido especial relevancia la acción desarrollada por Inditex en solidaridad con los afectados por la catástrofe causada por el buque petrolero Prestige. Tras una primera aportación de 270.000 euros realizada a Cruz Roja para atender a necesidades urgentes de materiales y equipos necesarios, la compañía destinó 6 millones de euros adicionales para colaborar en la resolución de los problemas ocasionados por el vertido contaminante.

\subsection{Otras formas de comunicación}

Existen otras formas no tan directas pero muy efectivas de comunicación a partir de la acumulación de outputs de las acciones diarias de la empresa, a través del contacto con sus clientes y con el aprendizaje continuo dentro de la organización ${ }^{12}$. Si un producto está bien diseñado y goza de una buena distribución, la empresa puede crear una buena reputación de marca sin ningún otro esfuerzo de comunicación directa. Zara, por ejemplo, es un caso emblemático: la ubicación, el

\footnotetext{
${ }^{12}$ Cerviño, J. (2002). Marcas internacionales. Cómo crearlas y gestionarlas. Pirámide. Madrid.
} 
diseño y el escaparatismo de sus tiendas, junto a un mix de producto innovador y siempre cambiante, así como una excelente relación calidad-precio, ha hecho del boca-oído su mejor forma de posicionar la marca en la mente de los consumidores ${ }^{13}$. También Loewe, la marca española más conocida en Japón, utiliza una estrategia de localización privilegiada para comunicar la marca en ese mercado. Esta firma sitúa sus locales dentro de grandes almacenes de prestigio, en las primeras plantas; así, el consumidor percibe que cualquier producto que se vende en estas tiendas es de lujo. Otros instrumentos como el precio, el packaging de la prenda o la manera de vestir del vendedor comunican algo al cliente potencial, y si se desea alcanzar un impacto de comunicación máximo, se debe cuidar la integración de todo el marketing mix de la moda y no de forma exclusiva el mix de comunicación.

\section{Conclusiones}

La comunicación de la moda es una política de marketing relativamente reciente y poco estudiada. Esta circunstancia parece estar justificada por la actualidad del término y porque todavía no existen unas sólidas bases bibliográficas ni un conjunto de autores especializados de referencia. La escasa fundamentación teórica de la disciplina dificulta encontrar una definición válida para esta investigación. Sin embargo, hemos comprobado cómo la dimensión comunicativa de la moda ha despertado un interés pluridisciplinar, aunque sin obtener resultados y conclusiones plenamente satisfactorios.

Lo que más interesa destacar en este artículo es la enorme cantidad de instrumentos de comunicación orientados a los diferentes públicos participantes en el mercado de la moda. Ello deviene en una industria singular desde un punto de vista comunicativo y exige a los emisores de este proceso una coherencia en la combinación de los

\footnotetext{
${ }^{13}$ Bonache, J. \& Cerviño, J. (1996). "Zara. El tejido internacional”, en Durán, J. J. y otros: Multinacionales españolas I: Algunos casos relevantes. Pirámide. Madrid.
} 
diferentes instrumentos que contribuyen a construir y a generar visibilidad en las marcas y operadores del sector.

\section{Bibliografía}

AA.VV. (2002). "Moda de España: punto de encuentro". Madrid, Sociedad Estatal para el Desarrollo del Diseño y la Innovación - DDI Ministerio de Economía.

ALET, J. (1994). Marketing Directo Integrado. Gestión 2000. Barcelona.

BONACHE, J. \& CERVIÑO, J. (1996). "Zara. El tejido internacional", en Durán, J. J y y otros: Multinacionales españolas I: Algunos casos relevantes. Pirámide. Madrid.

CERVIÑO, J. (2002). Marcas internacionales. Cómo crearlas y gestionarlas. Pirámide. Madrid.

CONTRERAS, M. (2002). "El nuevo templo de la moda" en revista Vogue Colecciones. Otoño/Invierno. Pág. 94-95.

FERNÁNDEZ SOUTO, A. B. (2002). Manual de programación e técnicas das rr.pp. Servizo de publicacións da Universidade de Vigo. Vigo.

FUNDACIÓ CETEMSA (2001). Resultados del estudio sobre el Impacto del Comercio Electrónico en el sector Textil Español. Edición propia. Barcelona.

HERRERO, M. (2004): "Fascinación a la carta: La moda en la postmodernidad" en CODINA, M. y HERRERO, M. (2004). Mirando la moda: once reflexiones. Ediciones Internacionales Universitarias. Madrid.

IVÁÑEZ, J. M. (2000). La gestión del diseño en la empresa. McGraw-Hill. Madrid.

LE MONNIER, F. (2000). Marketing Ferial. Gestión 2000. Barcelona. 